\title{
LA RELATIVISATION EN SYNTAXE FRANCAISE ET IGBO
}

\author{
Scholastica Ezeodili* \\ http://dx.doi.org/10.4314/og.v14i1.6
}

\section{Resume}

D'après Dubois et al, on appelle relativisation la formation d'une relative par une transformation qui enchâsse une phrase (phrase constituante) dans le syntagme nominal d'une autre phrase (phrase matrice) au moyen d'un relatif (409). Dans cette communication, nous avons examiné le processus de relativisation dans les deux langues: le français et l'igbo. Le processus est toujours explicitement repéré en français, ce qui n'est pas constamment le cas en igbo. Le présent travail a donc comme objectif de porter une réflexion sur le processus de relativisation dans les deux langues du français (langue étrangère) et de la langue igbo (langue nigériane du sud-est) et ensuite d'analyser trois des pronoms relatifs simples en français- que, qui, et dont, les plus fréquemment employés en discours oral et écrit, considéré comme problématiques aux étudiants étrangers. Notre approche qui est de nature descriptive est fondée sur la théorie de principes et paramètres de Chomsky. Cependant, la donnée de la présente étude est tirée des livres de grammaire desdites langues ainsi que des exercices écrits obtenus des apprenants finalistes de Nnamdi Azikiwe University, Awka. Le résultat de l'analyse a révèle que parmi les trois pronoms relatifs expérimentés, c'est surtout le pronom dont qui est le plus problématique.

\section{Abstract}

According to Dubois et al, relativization is the formation of a relative clause by a transformation that embeds a sentence (constituent sentence) into the noun phrase of another sentence (matrix sentence) by means of a relative pronoun (409). In this paper, we examined the process of relativization in French and Igbo languages. The process which is always explicitly marked in French is not always the case in Igbo. The present work therefore aims at discussing the process of relativization in the two languages of French and Igbo and then to analyze three of the simple relative pronouns in French, namely: que, qui, and dont, most frequently 
used in oral and written speech, considered problematic to the learners of French as a foreign language. The descriptive approach is based on Chomsky's theory of principles and parameters. However, the data in this study are drawn from the grammar books of these languages as well as written exercises of final year French students of Nnamdi Azikiwe University, Awka. The result of the analysis revealed that their difficulty lies mainly in the functioning of the relative pronoun dont.

\subsection{Introduction}

D'après Dubois et al dans le Dictionnaire de Linguistique, le terme «relativisation» désigne la formation d'une relative par une transformation qui enchâsse une phrase (phrase constituante) dans le syntagme nominal d'une autre phrase (phrase matrice) au moyen d'un relatif(409). Il s'agit d'un fusionnement de deux propositions dans un même environnement phrastique. Cette transformation qui se manifeste dans de diverses langues du monde, suivant la grammaire universelle de Chomsky, ne se réalise pas toujours du même processus. Dennis Creissels définit la relativisation comme :

Une opération logique de construction d'une propriété en utilisant un schéma phrastique exactement comme pour construire une phrase assertive, mais en laissant ouvert l'un des termes nominaux. Le terme nominal laissé ouvert dans le schéma phrastique relativisé (le terme qu'on peut faire apparaitre comme une variable dans la représentation logique) s’appelle terme relativisé.(15)

Ainsi, un élément dit pronom relatif est employé pour remplacer le terme nominal de la proposition relative.

L'objectif de cette communication qui se présente sur forme binôme se propose dans un premier lieu d'exposer d'une manière assez explicite, le processus de relativisation dans les deux langues du français (langue étrangère) et de la langue igbo (langue nigériane du sud-est) et ensuite d'analyser trois des pronoms relatifs simples en français- que, qui, et dont, les plus fréquemment employés en discours oral et écrit, considéré comme problématiques aux étudiants étrangers. Le travail est reparti en cinq sections. La deuxième partie est consacrée au cadre théorique, suivie par une 
Ezeodili : Syntaxe de Relativisation...

petite littérature de la relativisation. La syntaxe de la relativisation formera objet de la quatrième section. Ensuite, la méthodologie et l'analyse de données forment objet de cinquième chapitre qui aboutira enfin à la conclusion.

\subsection{Le Modele « Principe et Parametre » De Chomsky}

La Grammaire générative de Chomsky est en évolution continu et a abouti à plusieurs modèles et théories y compris le modèle principes et paramètres. Le point de vue des principes et paramètres est que la quasi-totalité des «connaissances linguistiques » dont l'homme fait montre n'est pas apprise mais plutôt partie intégrante de l'esprit humain. Mais alors, si une quelconque connaissance langagière est construite dans l'esprit de l'homme, cela doit être valable pour tous les humains et donc universelle. De ce point de vue, les langues ne sont pas si différentes qu'on le pense et les différences sont apparentes (ordre des mots dans l'énoncé, différences de mots, de sons,etc.) Les différences de surface sont ramenéesà un paramètre abstrait de variation dans le but de réduire les diverses options observées dans les grammaires individuelles. On impose des contraintes sur le système des règles des langues naturelles. Ces règles sont des principes universels de grammaire qui autorisent l'existence d'options pour les langues individuelles: ces options sont des paramètres de variation. Cette connaissance universelle se constitue de principes (des lois) qui fondent l'architecture de base de n'importe quel système linguistique, et de paramètres qui régissent les variations que pourrait manifester cette architecture.[www.ci/files/notes/seminaire\%20bogny\%20.]

Selon notre modèle, comme proposé par Chomsky, les deux éléments comme des concepts liés visent à tenir compte de l'invariance et des variations inter-linguistiques. Les principes expriment des contraintes universelles sur le langage humain alors que les paramètres définissent l'espace de variation interlinguistique. (L. Haegeman)

\subsection{Litterature de la Relativisation}

La relativisation en tant que phénomène grammatical est déjà discuté copieusement dans le discours. Les pronoms relatifs sont les éléments constitutifs du processus de la relativisation. D'une 
manière simple, c'est le fait de transformer en proposition relative [cnrtl.fr]

Mataebere a effectué un travail élaboré sur la relativisation en Igbo. D'aprèslui:

Igbo relative clauses are mainly realised by relativisationie a process whereby two sentences are collapsed into one with one of the sentences serving as an embedded structure and modifier to the subject NP (87).

La proposition relative se génère par relativisation, c.-à-d. un processus à travers lequel deux phrases sont fusionnées, l'un des phrases servant comme une structure enchâssée et modificateur du SN sujet (notre traduction).

Les études sur la relativisation (pronoms relatifs) dans des diverses langues sont déjà énormes. Monique Monville -Burston et Maria Kounouni ont observé que la relativisation demeure une zone morphosyntaxique problématique pour l'étudiant chypriote grec, jusqu'au niveau avancé. D'après elles, la situation diglossique complique les choses où ils ont à faire à deux niveaux de grec. De la même manière, BrunaScannone a présenté une étude sur la relativisation en Swahili visant àrepérer la structure de la proposition relative en Swahili sur le plan morphosyntaxique. Igbeneghu Boniface, de sa part, s'est donné la tache d'examiner la structure interne de la relativisation en français et puis de savoir si ce processus se fonde sur les mêmes principes et les mêmes paramètres dans quelques langues nigérianes - igbo, hausa et yoruba. Il a donné une explication du processus de la relativisation par un relativiseur ainsi :

D`ailleurs, la relativisation désigne la fusion entre deux structures phrastiques par moyen d'un relativiseur. La structure phrastique introduite par le relativiseur devient donc une proposition relative. La proposition relative modifie directement son antécédent qui, en général, est un syntagme déterminant (SD) dans la structure phrastique précédente.

Considérons, par exemple la phrase :

1. C'est une dame que j'admire. 
Ezeodili : Syntaxe de Relativisation...

Dans (1), que figure donc comme le relativiseur servant à modifier son antécédent une dame.

Alkhatib Mohammed observe le problème de distinction entre que et dont chez les apprenants arabophones qui produisent pour la plupart les énoncés comme *le film que j'ai parlé $/ *$ le film dont j'en ai parlé. Il observe comme source majeure de ce problème le fait qu'il n'y a qu'un seul pronom relatif en arabe pour exprimer les quatre pronoms relatifs que, qui, oùet dont dufrançais.

$\mathrm{Wu}$ a fait une étude aussi théorique que typologique de la relative dans les six langues à relative pre-nominale. Différentes langues du monde présentent les différentes stratégies de relativisation. On peut résumer jusqu'à ce point que le phénomène de relativisation est un fait universel mais qui se fonde aussi sur la syntaxe des diverses langues.

\subsection{Syntaxe de la Relativisation en Français et en Igbo}

Compte tenu de la définition de la relativisation comme processus d'enchâsser une proposition subordonnée par le biais d'un pronom relatif, sa représentation dans la phrase est impliquée. En français, le processus est complété par l'insertion d'un pronom relatif. Voyons les exemples suivants:

2a.La femme dont je parle est ma nièce.

2b. J'ai acheté une papaye qui est abimé.

2c. La papaye que j'ai achetée est abimée.

Ici, la subordonnée relative (ou la relative) est introduite par le pronom relatif dont, qui et que. Dans la phrase (2a), une fusion de deux propositions «La femme est ma nièce » et « je parle de la femme » a abouti àl'expression, « la femme dont je parle est ma nièce ». Ici, le relatif dont fonctionne comme complément de nom : (je parle de). Plus lucidement, on pourrait dire qu'il remplace un complément d'objet précédé par la préposition $d e$. Egalement, dans l'expression, "La maison dont il rêve coute très cher », Il rêve de cette maison.

Il importe donc de connaitre les verbes qui sont suivis par la préposition de. Le pronom relatif sert alors à remplacer un nom ou un pronom qui est placé avant lui. Elle sert aussi à introduire une proposition- proposition subordonnée. Dans les exemples 2(a-c), le 
pronom relatif permet de relier les deux propositions. En français, les formes du pronom relatifvarient ayant les formes simples et les formes composées. Les formes composées varient aussi en genre et en nombre ce qui pose tant de problèmes aux apprenants étrangers.

Ses formes simples comprennent: qui (généralement sujet), que (généralement objet direct), dont (qui assume toutes les fonctions introduites par de) On peut y ajouter le relatif adverbial où(MaugerG.163).

Notre travail ici abordera en particulier la syntaxe des formes simples: que, qui, dont. Le choix des formes simples réside sur le fait d'usage plus fréquent de ces formes simples dans le discours oral et écrit de tous les jours. Voyons quelques exemples à travers des phrases suivantes :

3a. Elle saisit la main que je lui tendais.

3b. La chat qu'iltraine appartient à son voisin.

3c. Mon frère a pris l'argent qui était dans le tiroir.

3d. Il a pris les fruits qui sont sur la table.

3e. C'est un document dont j'ai besoin.

3f. La maison dont je rêve se trouve au bord de la mer.

Le pronom relatif que fonctionne comme l'objet direct du verbe dans les phrases $3 \mathrm{a}, 3 \mathrm{~b}$. Le pronom relatif est exigé par les règles du français pour compléter le sens de la phrase. L'omission du relatif rendra agrammatical la dite phrase :

*Le chat il traîne appartient à son voisin.

Alors qu'en anglais le relatif peut se marquer par un morphème zéro (Emenanjo 211). Ainsi, la même phrase rendue en anglais peut signaler une absence du relatif :

The cat he is parading belongs to his neighbor.

Dans les phrases 3(c-d), le relatif qui est le sujet du verbe dans la proposition subordonnée.

De même, le pronom relatif simple dont s'emploie pour représenter un groupe du nom précédé de la préposition « de ». Dans la phrase $3(\mathrm{e}-\mathrm{f})$, la structure profonde avant la transformation : 
«J'ai besoin de ce document » et «Je rêve de cette maison ».

On observe également en français que le pronom relatif est postnominal du fait qu' il suit le nom tête.

La maison que j'ai achetée se trouve au bord de la mer. Nom tête REL SUB.

Selon Emenanjo, "In classical transformational grammar, relativization is the process of forming a relative clause construction." (211). En grammaire transformationnelle classique, la relativisation est le processus de formation d'une construction de la relative.[notre traduction]

En Igbo, comme c'est le cas dans d'autres langues comme le français et l'anglais, il occupe une position post nominale. Il n'existe pas de marqueur relatif ouvert en igbo puisqu'il n'existe ni pronom relatif ni adverbiaux. C'est plutôt un morphème tonal réalisé par "floating downstep tone" (Ndimele 2003 216), (Emenanjo, 1978 199).

D'ailleurs, lastratégie de relativisation en igbo implique la fusion de deux phrases simples. On la saisira mieux àtravers les exemples cidessous :

4 (a) Nwokē à bùnnà $m$ [Cet homme est mon père]

(b) Nwokē à nyèrègìakwà [Cet homme vous a donné un (des) œuf(s)]

5 (a) Nwokē à [Nwokē à nyèrègìakwà] bùnnà $m$.

(b) Nwokē à [Nwoke a-nyèrègìakwà] bùnnà $m$.

(c) Nwokē à [ønyēre gi akwà ]bùnnà m.

Imaginons que le $\mathrm{SN}$ sujet dans $4(\mathrm{a}-\mathrm{b})$ se marquent par un même référent comme observé en $5 \mathrm{a}$, la proposition $4 \mathrm{~b}$ est enchâssé comme proposition relative à l'intérieur du nom tête Nwokè à. Le nom tête fonctionne comme SN sujet de la proposition matrice. Suivant la référence identique, le SN sujet de la proposition enchâssé subit un effacement, aboutissant à la structure $5 \mathrm{~b}$. C'est important de remarquer ici que le SN effacé n'est pas substitue par un pronom relatif comme c'est le cas en français et en anglais. Il laisse plutôt un vide $(\varnothing)$. Ainsi, prédit- on d'emblée les difficultés des igbophones 
Ogirisi: a new journal of Africanstudies vol. 142018 dans l'apprentissage des pronoms relatifs français. La langue igbo se sert plutôt du changement duton.

Voyons quelques exemples de cette formulation tiré d'Emenanjo(2015)

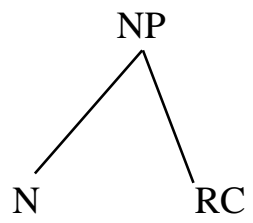

1. $\mathrm{O}$ zùtàrà igwē Igwè (HS) (machine)

2. $\mathrm{O}$ zùtàrà akwā akwà (HL) (cloth)

3. $\mathrm{O}$ zùtàrà àkwa àkwa(LH) (egg)

4. $\mathrm{O}$ zùtàrà àkwa àkwà(LL) (bed) māra mmā She bought a beautiful machine (She bought a machine which is good) mārammā She bought a beautiful cloth (She bought a cloth which is beautiful)

mārammā She bought good eggs

( She bought eggs which are good)

mārammā She bought a beautiful bed She bought a bed which is beautiful

Le relatif en igbo fonctionne comme sujet modificateur de la proposition relatif si le verbe suit immédiatement le SN modifié.

Uchè zùrù akwā $\emptyset$ dārā oke ọnụ [Uche a acheté un habit qui est très cher]

Dans le cas de modificateur sujet du relatif, le ton se réduit àun «steptone »

Dans le cas du modificateur relatif objet « object modifying relative clause » le verbe de la proposition relative est toujours marqué par le ton bas sans tenir compte du ton inhérent du verbe. L'effacement s'effectue au niveau du SN objet de la proposition relative. :

La relativisation se produit en Igbo aussi par le processus de l'enchâssement d'une phrase à une autre. En ce sens, les phrases déclaratives :

Àdaóbi bù ényì m

(Adaobi etre ami poss moi)

Adaobi est mon amie

et

Àdaóbi abiala. 
Ezeodili : Syntaxe de Relativisation...

(Adaobi passe (perfectif) venir)

Peuvent se relativiser comme suit :

Adaobi nke bu enyi m abiala.

(Adaobi REL- être amiposs.passe arriver)

Adaobi qui est mon ami est arrivé

En général, les formes du pronom relatif ne sont pas toujours marquées ouvertement en Igbo. C'est plutôt la tonalisation du verbe à un mode fini, ainsi pour relativiser une phrase simple, le ton bas inhérent du verbe est élevé au ton haut. On le saisira mieux dans la phrase :

Atụrụahụ tara ji di ime

(moutonce-laRel passe manger igname est enceinte)

Le mouton qui a mangé l'igname est enceint.

La relativisation se veut un processus de construire des phrases complexes dans chacune des dites langues. Le processus comprend toujours l'enchâssement d'une proposition subordonnée dans une phrase matrice.Le relatif est post-nominal dans les deux langues du fait qu'il suit le nom tête. La divergence se marque suivant que le processus est ouvertement marqué en français par le pronom relatif maismarqué en igbo par le ton.

\subsection{Méthodologie}

Les données de l'étude sont recueillies des exercices écrits proposés aux 30 étudiants finalistes de UNIZIK (six males et 24 femelles) basées sur les trois formes simples du pronom relatif -qui, que, dontce afin de déterminer leur niveau de compétence dans l'emploi des relatifs simples. Pour ce faire, deux groupes de tests sont impliqués : Pour le premier groupe de test, comprenant 20 propositions, ils devraient choisir le pronom le plus convenable pour remplir les phrases; La deuxième section consiste à rendre en français 10 groupes de questions dont les trois pronoms relatifs sont encore engagés. La méthode d'analyse est le simple pourcentage. 


\section{Analyse}

Résultat par un simple pourcentage-Test de remplissage

\begin{tabular}{|l|l|l|l|}
\hline & $\begin{array}{l}\% \\
\text { réponses de } \\
\text { correctes }\end{array}$ & $\begin{array}{l}\text { \% de réponses } \\
\text { incorrectes }\end{array}$ & $\begin{array}{l}\text { Forme du } \\
\text { pronom } \\
\text { relatif } \\
\text { correcte }\end{array}$ \\
\hline 1 & $73.3 \%$ & $26.6 \%$ & Qui \\
\hline 2 & $40 \%$ & $60 \%$ & Dont \\
\hline 3 & $86.6 \%$ & $13.3 \%$ & Que \\
\hline 4 & $60 \%$ & $40 \%$ & Qui \\
\hline 5 & $60 \%$ & $40 \%$ & Que \\
\hline 6 & $40 \%$ & $60 \%$ & Dont \\
\hline 7 & $86.6 \%$ & $13.3 \%$ & Qui \\
\hline 8 & $86.6 \%$ & $13.3 \%$ & Que \\
\hline 9 & $73.3 \%$ & $26.6 \%$ & Dont \\
\hline 10 & $86.6 \%$ & $13.3 \%$ & Qui \\
\hline 11 & $46.6 \%$ & $53.3 \%$ & Dont \\
\hline 12 & $46.6 \%$ & $53.3 \%$ & Dont \\
\hline 13 & $46.6 \%$ & $53.3 \%$ & Que \\
\hline 14 & $66.6 \%$ & $33.3 \%$ & Qui \\
\hline 15 & $26.6 \%$ & $73.3 \%$ & Dont \\
\hline 16 & $73.3 \%$ & $26.6 \%$ & Qui \\
\hline 17 & $40 \%$ & $53.3 \%$ & Dont \\
\hline 18 & $20 \%$ & $80 \%$ & Dont \\
\hline 19 & $80 \%$ & $20 \%$ & Qui \\
\hline 20 & $66.6 \%$ & $33.3 \%$ & Que \\
\hline & & & \\
\hline
\end{tabular}

Résultat de l'exercice 2 -Exercice de traduction

\begin{tabular}{|l|l|l|}
\hline $\begin{array}{l}\text { Numéro de } \\
\text { proposition }\end{array}$ & $\begin{array}{l}\% \text { de traductions } \\
\text { correctes }\end{array}$ & $\begin{array}{l}\text { Forme correcte du } \\
\text { pronom relatif }\end{array}$ \\
\hline 1 & 80 & Que \\
\hline 2 & $26.6 \%$ & Dont \\
\hline 3 & $86.6 \%$ & Que \\
\hline 4 & $73.3 \%$ & Qui \\
\hline 5 & $53.3 \%$ & Dont \\
\hline 6 & 93.3 & Que \\
\hline 7 & $60 \%$ & Que \\
\hline
\end{tabular}


Ezeodili : Syntaxe de Relativisation...

\begin{tabular}{|l|l|l|}
\hline 8 & $40 \%$ & Qui \\
\hline 9 & $60 \%$ & Que \\
\hline 10 & $33.3 \%$ & Dont \\
\hline
\end{tabular}

- D'après l'analyse des réponses des étudiants, on a constaté que c'est surtout le pronom dont qui pose le plus du problème. Un grand nombre n'a pas pu rendre la forme correcte du pronom dont. Donc, seul $40 \%$ ont pu produire la forme correcte. alors que $45 \%$ répondent avec le pronom que ainsi : *Le prisonnier que je t'ai parlé s'est évadé. Ceci peut s'expliquer par l'interférence négative de l'anglais: The lady that I spoke about... Le cas de la réponse erronée de qui également:*Vous verrez les choses que vous serez très fiers au lieu de vous verrez. les choses dont vous serez très fiers.

- La phrase anglaise la plus problématique à rendre en français: The child she is looking after is not her own (26.6\%). Les phrases réalisées: L'enfant qu'elle s'occupe....au lieu de :l'enfant dont elle s'occupe....; * Le prisonnier que je t'ai parlé s'est évadé. (sur généralisation) *Vous verrez des choses que vous serez très fiers.Ceci pourrait s'attribuer à la structure anglaise qui accepte également le phénomène de preposition stranding. Ainsiproduit-on des énoncés comme: The prisoner that I spoke to you about, the child that she is looking after........

\subsection{Conclusion}

L'apprentissage de la grammaire de langue étrangère ne cesse d'être un point problématique pour les apprenants étrangers. Le cas devient pire surtout dans les langues dont le point grammatical exposé est absent. Or c'est le cas ici du pronom relatif qui n'est pas ouvertement réalisé en igbo mais qui est impliqué des fois avec variation de ton ou position du SN tête. Nous avons constatéé travers les exercices de classe que c'est surtout le pronom dont qui se projette comme le plus problématique. Ce phénomène impliquant la compétence dans le fonctionnement du verbe transitive indirecte qui subit des transformations demande donc une attention particulière dans la classe de langue.

Pour un résultat plus adéquat, nous proposons une combinaison de l'approche déductive et inductive suivie d'unesérie d'exercices. Il 
Ogirisi: a new journal of Africanstudies vol. 142018

faut tout d'abord identifier les problèmes avant de passer aux exercices de systématisation. En plus, la nouvelle approche favorise le travail en groupe.

La classe de langue devrait être assez équipée avec des gadgets technologiques modernes afin que les apprenants puissent agir et interagir naturellement dans l'environnement. Pour ce faire, nous recommandons l'emploi du projecteur et tableau blanc interactif dans la classe de langue.

*Scholastica Ezeodili, PhD, Department of Modern European

Languages, Nnamdi Azikiwe Universty Awka Anambra State daccord2014@gmail.com/su.ezeodili@unizik.edu.ng 
Ezeodili : Syntaxe de Relativisation...

\section{CEUVRES CITEES}

Alain Lemarechal. «Typologie des relatives et théories de la relative » $\operatorname{Lin} x$ [en ligne],11/ 1999, mis en ligne le 29 juin 2012, consulté le 17 Octobre 2017. URL : http://linx.revues.org/888;D0I :10.4000/linx.888.

Alkhatib Mohammed «Traitement de l'erreur grammaticale en français langue étrangère » https://academia.edu/7928429/Traitements_de_I_erreur_gra mmaticale, no date

Creissels, D.Cours de syntaxe générale. Paris : Presses universitaires de France, 2004.

Dubois, Jean et al Dictionnaire de Linguistique et des sciences du langage. Paris: Larousse, 1994.

EmenanjoNolue E.A Grammar of Contemporary Igbo.(students' edition). Port Harcourt: M \& J Grand Orbit CommunicationsLtd; 2015.

Haegemon, L. "Linguistics: Theory of Principles and parameters"International Encyclopedia of the Social \& Behavioral Sciences. 2001 (8957-8961) sciencedirect.com.

Ibeneghu Boniface «Quelques remarques sur la syntaxe de la relativisation en français» The Journal of International Social Research. Cilt : 7 Sayi :33 Volume :7 Issue :33 [www.sosyalarastirmalar.com/cilt7/sayi33_pdf/1di...]

Monique Monville-Burston et Maria Kounomi'Deux procedures experimentales pour tester la relativisation dans l'interlangue d'apprenants chypriotes hellénophones en FLE' enl.auth.gr.consulte 29/4/2017.

Mataebere ,Iwundu. 'Igbo Relative clauses : some general aspects of language 'Nsukka journal of linguistics and African languages.1(1978):87-99.

Monique Monville-Burston et Maria Kounomi «Deux procédures expérimentales pour tester la relativisation dans l'interlangue d'apprenants chypriotes hellénophones en FLE » enl.auth.gr. consulté 29/4/2017.

$\mathrm{Wu}$ Tong'La relativisation prénominale Étude comparative sur l'amharique, le basque, le chinois mandarin, le japonais, le quechua et le turc “ Mémoire présenté pour l'obtention du grade 
de Master ès sciences humaines en sciences du langage «Le modèle chomskyen de la description linguistique: Des principes et Paramètres au Programma Minimaliste» Equipe de Recherche Théories \& Modèles linguistiques/ Séminaire sur la Grammaire Générative/ aout 2007/Dr BognyYapo J. /ILA/ Dep. Des Sciences du Langage bogny@atp2.net/jbogny@ucocody.ci

. [www.ci/files/notes/seminaire\%20bogny\%20..]

\section{ANNEXE}

\section{EXERCICE ECRIT}

\section{Remplissez avec qui, que ou dont}

1. Mon frère a pris l'argent ----était sur le tiroir.

2. Le prisonnier ----je t'ai parlé s'est évadé.

3. J'ai soigneusement range la robe----j'ai achetée.

4. Au cinéma, j'ai vu un film----m'a beaucoup plu.

5. J'ai verséà la banque l'argent ---tu m'avais remis.

6. Vous verrez des choses ----vous serez très fiers.

7. Les enfants ont cassé le vase ---sur la table.

8. Est-ce que tu sais où est le magazine ---j'ai acheté hier ?

9. Je ne sais pas où trouver les renseignements---j' ai besoin..

10. La personne ---m'a contacté hier était intéressé par mon CV.

11. La maison---les volets sont verts appartient a mon oncle.

12. C'est le film ----tout le monde parle en ce moment.

13. J'ai reçu un message ---j'ai du mal a comprendre.

14. Le paquet ---il a reçu hier venait des Etats-Unis.

15. N'ouvrez pas les pièces jointes---tu ne connais pas.

16. Tous ceux qui l'ont rencontrée se souviennent d'elle.

17. Quel est le cadeau ---tu as le plus envie ?

18. Le vocabulaire---je me souviens le mieux est .....

19. Les clients ---sont arrives hier repartent demain.

20. La fille---j'aime ne m'aime pas.

\section{TRADUIRE EN FRANÇAIS}

It is a game that I like. 2 The child she is looking after is not her own. 3.I have lost the pen which she gave me. 4 Did you take the paper which was there? 5. Do you know the man that lives here ? 6. I am not interested in the people you are talking about. 7. I bought the dress which you recommended. 8. I know many languages that are spoken in Europe. 9. The lady I saw is very beautiful. 10. That is the girl I told you about. 